\title{
Influence of Oxyfluorination on Physicochemical Characteristics of Carbon Fibers and their Reinforced Epoxy Composites
}

\author{
Min-Kang Seo and Soo-Jin Park* \\ Department of Chemistry, Inha University, Incheon 402-751, Korea
}

Received September 10, 2008, Revised October 30, 2008; Accepted October 31, 2008

\begin{abstract}
The effect of oxyfluorination temperature on the surface properties of carbon fibers and their reinforced epoxy composites was investigated. Infrared (IR) spectroscopy results for the oxyfluorinated carbon fibers revealed carboxyl/ester $(\mathrm{C}=\mathrm{O})$ and hydroxyl $(\mathrm{O}-\mathrm{H})$ groups at 1632 and $3450 \mathrm{~cm}^{-1}$, respectively, and that the oxyfluorinated carbon fibers had a higher O-H peak intensity than that of the fluorinated ones. X-ray photoelectron spectroscopy (XPS) results indicated that after oxyfluorination, graphitic carbon was the major carbon functional component on the carbon fiber surfaces, while other functional groups present were $\mathrm{C}-\mathrm{O}, \mathrm{C}=\mathrm{O}, \mathrm{HO}-\mathrm{C}=\mathrm{O}$, and $\mathrm{C}-\mathrm{F}_{\mathrm{x}}$. These components improved the impact properties of oxyfluorinated carbon fibers-reinforced epoxy composites by improving the interfacial adhesion between the carbon fibers and the epoxy matrix resins.
\end{abstract}

Keywords: oxyfluorination, surface properties, carbon fibers, impact properties, interfacial adhesions.

\section{Introduction}

Carbon fiber-reinforced composites are a class of advanced materials that have been developed for a variety of hightechnology applications in the aerospace, automotive, and sporting goods industries. However, in order to obtain the advantages of the excellent mechanical properties of carbon fibers in composites, optimized interfacial adhesion between fiber and matrix is necessary. In view of the fact that stress is transferred from one fiber to another through the matrix, the interface plays a major role in the overall mechanical performance of carbon fiber-reinforced composite materials. ${ }^{1-3}$ It is the efficacy of adhesion between the reinforcing fibers and the matrix that determines the amount of load, transferred through shear that can be distributed among the fibers, ultimately determining the maximum load-bearing capacity of composite structures. ${ }^{4}$

To improve fiber/matrix adhesion, it is necessary to increase the surface polarity, create more sites for hydrogen bonding and improve the possibility for mechanical interlocking between the fiber materials and the surrounding polymer matrix, which leads to better stress transfer from the matrix materials to the filling fiber ones. ${ }^{5-10}$

However, the means of improving fiber/matrix adhesion for a given surface treatment remain less than well developed or understood. Therefore, in the present study, direct oxyfluorination is applied in order to modify the surface characteristics of carbon fibers. Notably, oxygen gas is used

*Corresponding Author. E-mail: sjpark@inha.ac.kr in place of an HF catalyst for oxyfluorination of the fibers. The relationship between the oxygen content and the composites' fiber/matrix adhesion is investigated using a combination of surface analyses and impact tests.

\section{Experimental}

Materials. The carbon fibers were untreated and unsized polyacrylonitrile (PAN)-based high strength fibers, TZ-307 (12 K), manufactured by Taekwang of Korea. Their average diameter was approximately $7 \mathrm{~mm}$, and their typical tensile strength and modulus were about 3.5 and $245 \mathrm{GPa}$, respectively. The matrix employed was an epoxy resin based on diglycidyl ether of bisphenol A (DGEBA, YD-128, Kukdo Chem. Co.), which had an epoxide equivalent weight of $187{\mathrm{~g} . \mathrm{eq}^{-1}}^{-1}$ and a viscosity of about $5,000 \mathrm{cps}$ at $25^{\circ} \mathrm{C}$. Diaminodiphenylmethane (DDM) was selected for use as a hardener.

Sample Preparation. The carbon fibers were subjected to oxyfluorination under different conditions. The oxyfluorination reaction was performed in a batch reactor made of nickel with an outer electric furnace, as shown in Figure 1. After evacuation, the fluorine and oxygen mixtures $\left(\mathrm{F}_{2} / \mathrm{O}_{2}\right.$ gases) were introduced to the reactor at room temperature, and then the reactor was heated to the treatment temperature. After the reaction, the specimens were cooled to room temperature, after which the reactive gases were purged from the reactor with nitrogen. In the case of a room-temperature reaction, the reactor was cooled and evacuated in a cooling bath prior to the charging of fluorine. After purging the fluorine with nitrogen, the reactor was removed from 


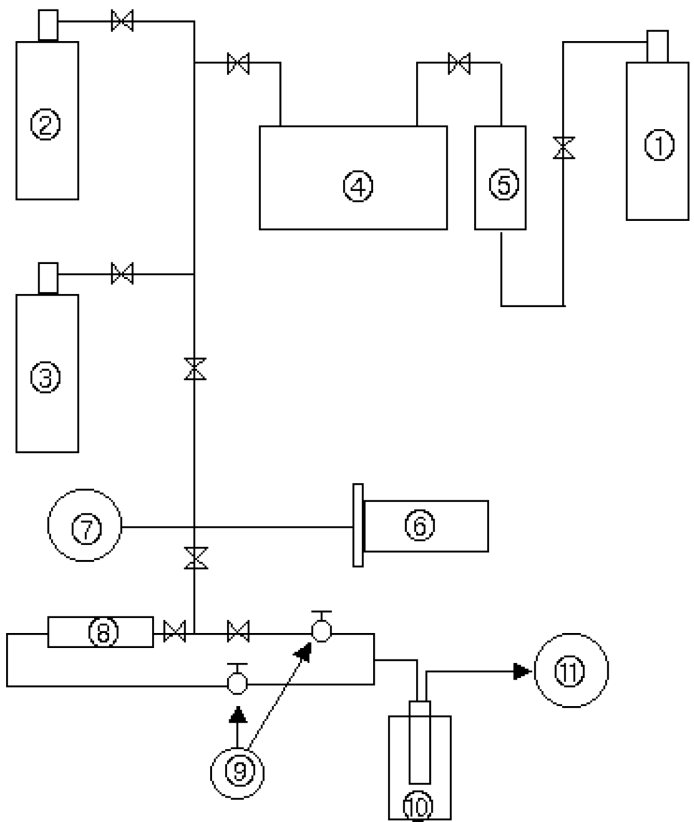

Figure 1. Schematic diagram of oxyfluorination reactor: (1) $F_{2}$ gas cylinder, (2) $\mathrm{N}_{2}$ gas cylinder, (3) $\mathrm{O}_{2}$ gas cylinder, (4) Buffer tank, (5) $\mathrm{HF}$ absorber (NaF pellet), (6) Reactor, (7) Pressure gauge, (8) $\mathrm{F}_{2}$ absorber $\left(\mathrm{Al}_{2} \mathrm{O}_{3}\right)$, (9) Glass cock, (10) Liquid nitrogen, (11) Rotary vacuum pump.

Table I. Experimental Oxyfluorinated Conditions of PAN-Based Carbon Fibers Employed

\begin{tabular}{ccc}
\hline Specimens & $\mathrm{F}_{2} / \mathrm{O}_{2}$ Mixtures $(\%)$ & Oxyfluorination Temperature $\left({ }^{\circ} \mathrm{C}\right)$ \\
\hline as-received & - & - \\
\hline CFO-RT & & 25 \\
CFO-100 & $50 / 50$ & 100 \\
CFO-300 & & 300 \\
CFO-400 & & 400 \\
\hline
\end{tabular}

the cooling bath. The total gas pressure was $0.2 \mathrm{MPa}$ and the nominal reaction time was $10 \mathrm{~min}$ at the treatment temperature. Table I listed the experimental conditions of the carbon fibers studied.

Unidirectional composite laminates were conventionally prepared by continuous impregnation of fibers using a drum-winding technique with subsequent hot pressing. The laminates, composed of 32 prepreg plies, were 7.4 MPa hotpressed at $150{ }^{\circ} \mathrm{C}$ for 150 min by means of a vacuum bagging method. The fiber volume fraction of the bulk specimens was about $52 \%( \pm 0.2 \%)$ for all of the composites.

Measurements. The occurrence of surface chemical reactions on the oxyfluorinated carbon fiber surfaces was also investigated. Samples of specimens that were selected from among the fibers subjected to varying oxyfluorination treatments were loaded onto $\mathrm{KBr}$ disks (0.5 mg sample on 200 $\mathrm{mg} \mathrm{KBr}$ ) for the purposes of Fourier transform infrared
(FTIR) measurements by Digital FTS-80 (Bio-Rad).

The surface $\mathrm{pH}$ of oxyfluorinated carbon fibers was measured by pH-meter (HI 9210 N, Hanna Instrument Co.) according to the ASTM D 3838. About $1.0 \mathrm{~g}$ of each sample was added to $20 \mathrm{~mL}$ distilled buffer solution ( $\mathrm{pH} 7.0,25^{\circ} \mathrm{C}$ ), and the mixture was shaken for $12 \mathrm{~h}$. The $\mathrm{pH}$ was measured after filtration with a nylon membrane filter $(0.45 \mu \mathrm{m}$ in diameter).

The acid value of oxyfluorinated carbon fibers was determined by Boehm's titration technique. ${ }^{11}$ In the case of an acidic value, about $1.0 \mathrm{~g}$ of sample was added to $100 \mathrm{~mL}$ of $0.1 \mathrm{~N} \mathrm{NaOH}$ solutions and shaken for $24 \mathrm{~h}$. After filtration with a nylon membrane filter $(0.45 \mu \mathrm{m}$ in diameter $)$, the mixture was titrated with $0.1 \mathrm{~N} \mathrm{HCl}$ standard solutions, using $0.01 \mathrm{M}$ phenolphthalein solution as an indicator.

X-ray photoelectron spectroscopy (XPS) measurement of the fiber surfaces was performed using a VG Scientific ESCA LAB MK-II spectrometer equipped with an $\mathrm{Mg}-\mathrm{K} \alpha$ $\mathrm{X}$-ray source. The base pressure in the sample chamber was maintained within the $10^{-8}$ to $10^{-9}$ Torr range.

A low-velocity impact test was conducted using a dropweight impact tester (ROSAND Instrumented Falling Weight Impact Tester, Type 4). The maximum drop height was $1 \mathrm{~m}$, from which a maximum velocity of $1.8 \mathrm{~m} / \mathrm{sec}$ was attained.

\section{Results and Discussion}

An FTIR spectroscope is applied to observe the effect of oxyfluorination temperature on the carbon fiber surfaces. Figure 2 shows the FTIR results of oxyfluorinated carbon fiber specimens. Carboxyl/ester groups $(\mathrm{C}=\mathrm{O})$ at $1632 \mathrm{~cm}^{-1}$ and hydroxyl groups $(\mathrm{O}-\mathrm{H})$ at $3450 \mathrm{~cm}^{-1}$ are observed. Especially, the O-H group of the fibers exhibits the highest intensity at the oxyfluorination temperature of $100^{\circ} \mathrm{C}$. This kind of result can affect the surface characteristics of carbon fibers, leading to an increase of specific polarity and the for-

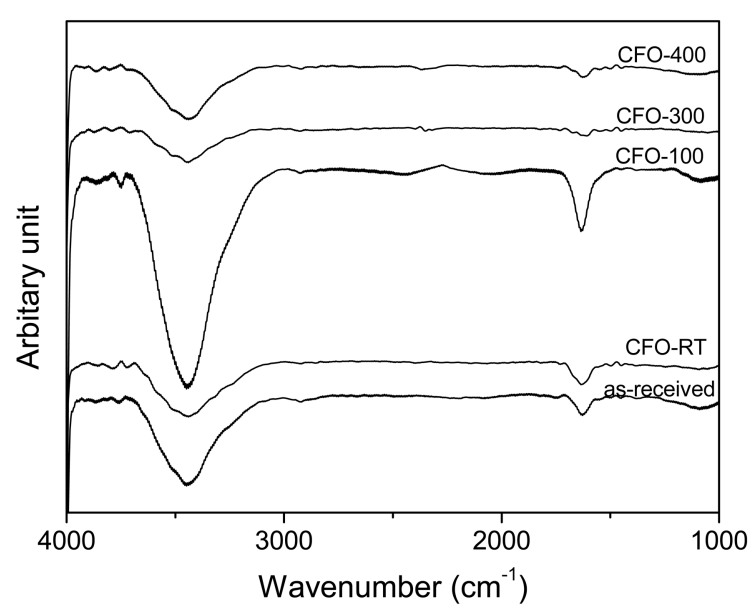

Figure 2. FTIR spectra of oxyfluorinated carbon fibers as a function of oxyfluorination temperature. 
Table II. pH, Acid Value and Compositions of Surface Oxyfluorinated Carbon Fiber Samples

\begin{tabular}{ccccccc}
\hline Specimens & $\mathrm{pH}$ & Acid Value [meq. $\left.\mathrm{g}^{-1}\right]$ & \multicolumn{3}{c}{ XPS [at\%] } \\
\cline { 4 - 7 } & & & $C_{1 s}$ & $F_{1 s}$ & $O_{1 s}$ & $O_{1 s} / C_{1 s}$ \\
\hline as-received & 7.02 & 15.0 & 88.4 & - & 9.6 & 0.11 \\
CFO-RT & 6.95 & 18.5 & 74.2 & 13.6 & 10.9 & 0.15 \\
CFO-100 & 6.94 & 22.1 & 67.7 & 19.5 & 11.3 & 0.17 \\
CFO-300 & 6.94 & 20.3 & 79.9 & 10.6 & 10.3 & 0.13 \\
CFO-400 & 6.95 & 18.4 & 76.9 & 8.9 & 10.1 & 0.13 \\
\hline
\end{tabular}

mation of H-bonding. ${ }^{12-15}$

Table II lists the $\mathrm{pH}$ and acid values of oxyfluorinated carbon fibers. The results exhibit that the surface of oxyfluorinated carbon fibers shows a slight excess of acidic properties. This is probably due to the formation of acidic groups, such as carboxyl, carbonyl and hydroxyl group, on the carbon fiber surfaces, resulting from the use of oxidizer for oxyfluorination.

Figure 3(a) shows narrow scan spectra of the $C_{1 s}$ region of
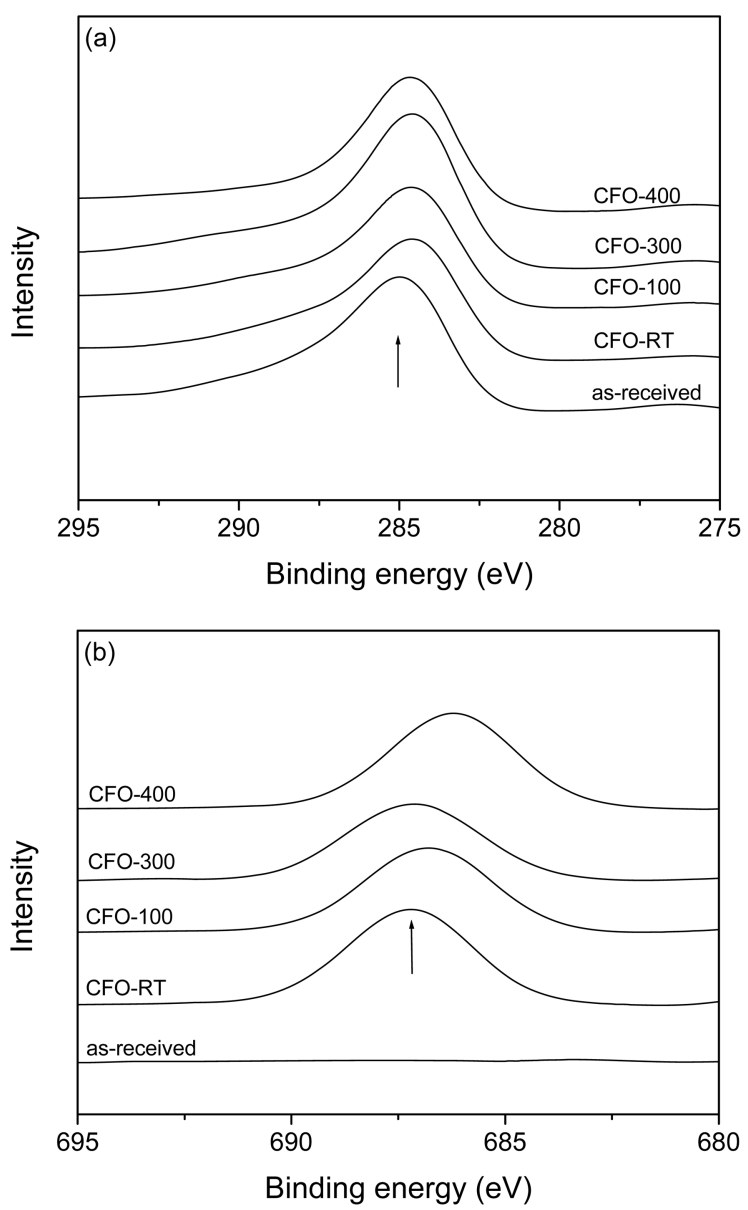

Figure 3. $C_{1 s}$ and $F_{1 s}$ narrow scan XPS spectra of oxyfluorinated carbon fibers: (a) $C_{1 s}$ spectra and (b) $F_{1 s}$ spectra. the oxyfluorinated carbon fibers. The binding energy $\left(E_{b}\right)$ of the $C_{1 s}$ peak for the as-received carbon fibers is $285 \mathrm{eV}$, representing the most graphitic carbons (C-C), which undergoes a slight shift towards a lower $E_{b}$ owing to a lowering of the Fermi level $\left(E_{F}\right)$ and a subsequent decrease of the energy gap between the $C_{1 s}$ core level and $E_{F}{ }^{16}$ The difference between the FWHM values for the as-received and the CFO-RT is $0.65 \mathrm{eV}$. A higher FWHM value is found for the CFO-100 sample, presumably due to the surface oxyfluorination. Figure 3(b) shows narrow-scan spectra of the $F_{1 s}$ region of the oxyfluorinated carbon fibers. Due to the surface oxyfluorination, a higher FWHM value as well as a shoulder in the higher binding energy range of 686.7 688 \pm 0.1 $\mathrm{eV}$ is observed for the oxyfluorinated carbon fiber specimens.

Typical XPS spectra of the $C_{1 s}$ peak region at $285 \mathrm{eV}$ deconvoluted into surface functional group contributions of the oxyfluorinated carbon fiber specimens are shown in Figure 4(a). It is found that the $C_{1 s}$ peaks of the CFO-100 specimen can be fitted to four line shapes with binding energies at $284.8,284.7,286.9$ and $288.8 \mathrm{eV}$. These different binding energy peaks are assigned to center at $284.8 \mathrm{eV}, \mathrm{C}-\mathrm{C}$ at $284.7 \mathrm{eV},-\mathrm{C}=\mathrm{O}$ at $286.9 \mathrm{eV}$ and $\mathrm{HO}-\mathrm{C}=\mathrm{O}$ at $288.8 \mathrm{eV}$. It can thus be noted that two functional components are present on the carbon fiber surfaces in addition to the C-C peak after oxyfluorination, which is located at $285.0 \mathrm{eV}$. Figure $4(\mathrm{~b})$ shows the $F_{1 s}$ peak deconvolution of the oxyfluorinated carbon fibers in the $687.0 \mathrm{eV}$ regions. In the $F_{1 s}$ spectrum the physical component occurs at $E_{b}=686.7 \pm 0.05 \mathrm{eV}$. That is, two components at $687.1 \pm 0.03 \mathrm{eV}\left(C F_{1}\right)$ and at 686.13 $\pm 0.04 \mathrm{eV}\left(C F_{2}\right)$ in $F_{1 s}$ spectra are assigned to physical C- $F_{x}$ $(1<x<2)$ groups present on the surface of the fibers or at structural defects.

Quantitative peak analysis is also carried out to determine the surface element concentrations. The resulting surface element concentrations of the oxyfluorinated carbon fiber specimens are listed in Table II. It is found that the surface carbon concentrations of the as-received and CFO-100 carbon fiber specimens are $88.38 \mathrm{at} \%$ and $67.72 \mathrm{at} \%$, respectively. A lower surface carbon concentration in the CFO100 carbon fiber specimen compared to the as-received one could be attributed to the bonding of oxygen or fluorine on 

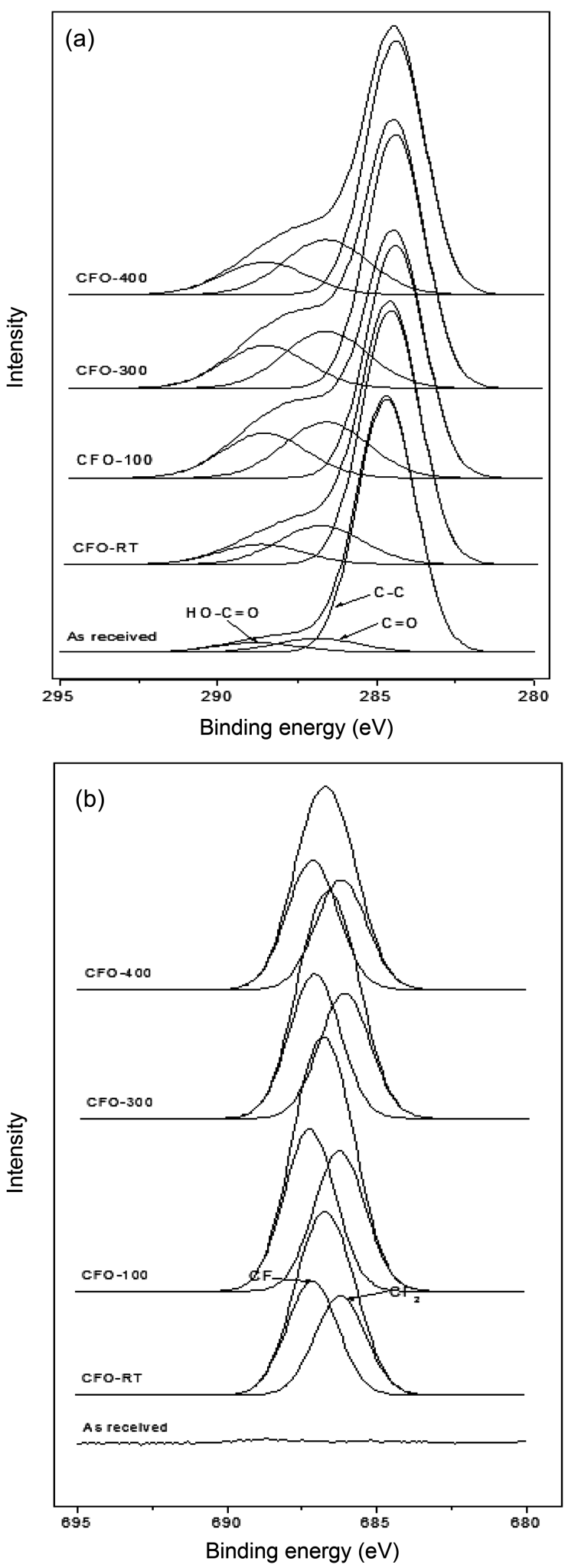

Figure 4. High-resolution curve fitted $C_{1 s}$ and $F_{1 s}$ peaks of the oxyfluorinated carbon fibers: (a) $C_{1 s}$ spectra and (b) $F_{1 s}$ spectra.

the carbon fiber surfaces produced by the oxyfluorination. The surface fluorine concentration ranges of the oxyfluori- nated carbon fiber specimens are 8.91 19.50 at $\%$. The higher fluorine concentration on the CFO-100 specimen $(19.50 \mathrm{at} \%)$ surfaces can be also attributed to the surface oxyfluorination of the fibers.

Consequently, with increasing the amount of fluorination on the surface, the content of graphite-type carbon decreases, whereas the relative amounts of $\mathrm{C}-F_{x}$ increases with increasing the fluorination temperature and oxygen content, as expected, which is probably due to the kinetic reasons and moreover, the fluorination of the bulk phase of the fiber can be considered. ${ }^{17,18}$

It is now well known that localized impact loading on fibers-reinforced composites frequently generates extensive damage that can, under certain circumstances, result in a significant reduction in the load-bearing capability of the structure. The impact strength is one of the principle mechanical properties in order to evaluate the degree of toughness in composite materials. When such materials are impacted, the transferring load is absorbed into them, affecting the residual mechanical properties. ${ }^{19}$

The total impact energy measured, $E_{t}$, is the sum of the initiation energy, $E_{i}$, and the propagation energy, $E_{p}$. If the material behavior is elastic to failure and the stress in the specimen is adequately described by simple beam theory, then the initiation energy in a unidirectional composite reinforced with one type of fiber is given by follow equation. ${ }^{20}$

$$
E_{i}=\frac{L w t}{18} \cdot v_{f} \cdot \frac{\sigma_{f}^{2}}{E_{f}}
$$

where $L$ is the span length, $w$ the specimen width, $t$ the specimen thickness, $v_{f}$ the fiber volume fraction, $\sigma_{f}$ and $E_{f}$ the fiber stress and the fiber modulus at failure by tension or compression, respectively.

The second portion of the impact energy, $E_{p}$, contributes in a more complex way, since it is dependent on material fracture mechanisms that, in turn, are influenced by specimen geometry as well as by material properties. For specimens having a similar geometry, the relative percentage of energy absorbed in fracture initiation and propagation provides an indication of the ductility of the materials. The ductility index (DI) can be defined as the ratio of propagation energy to initiation energy. ${ }^{21}$

$$
D I=\frac{E_{p}}{E_{i}}
$$

Therefore, brittle materials have low $E_{p}$ and, as a result, low $D I$, as deduced from above equation.

Figure 5 shows the impact strengths of the oxyfluorinated carbon fibers-reinforced composites, measured using the drop weighting impact tester. The impact properties of the composites are improved by increasing the fluorine content, which finding correlates with the fracture toughness results. That is, the composites oxyfluorinated with mixed $\mathrm{F}_{2} / \mathrm{O}_{2}$ gases, compared with those lacking oxyfluorination, were 


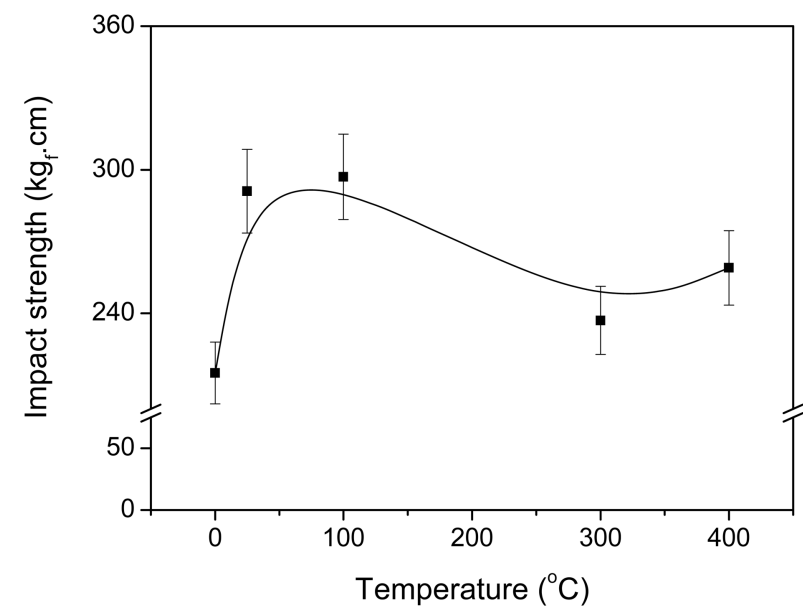

Figure 5. Impact strength of oxyfluorinated carbon fibers-reinforced composites as a function of oxyfluorination temperature.

much toughened in resistance to impact-damage. In the course of inducing low-velocity $\left(1.8 \mathrm{~m} \cdot \mathrm{s}^{-1}\right)$ impact-damage to the composites, the damage zone acts as a soft region within a stiff laminate, serving to magnify stress locally by means of matrix cracking, fiber debonding, delamination, and fiber fractures. ${ }^{22}$ These impact-loading-induced failure behaviors are hindered by the presence of hydrogen between fiber surfaces and matrix resins in the composites. That is, this hydrogen bonding, by means of the increase in the specific or polar component, interrupts the formation of crackgrowth paths among fibers, matrix and fillers, resulting in improved fracture toughness of the composites.

Impact energy is the principle mechanical properties in order to evaluate the degree of toughness in composite materials. When such materials are impacted, the transferring load is absorbed into them and affecting the residual mechanical properties. ${ }^{23}$ The $E_{p}, E_{i}$, and $D I$ results for the

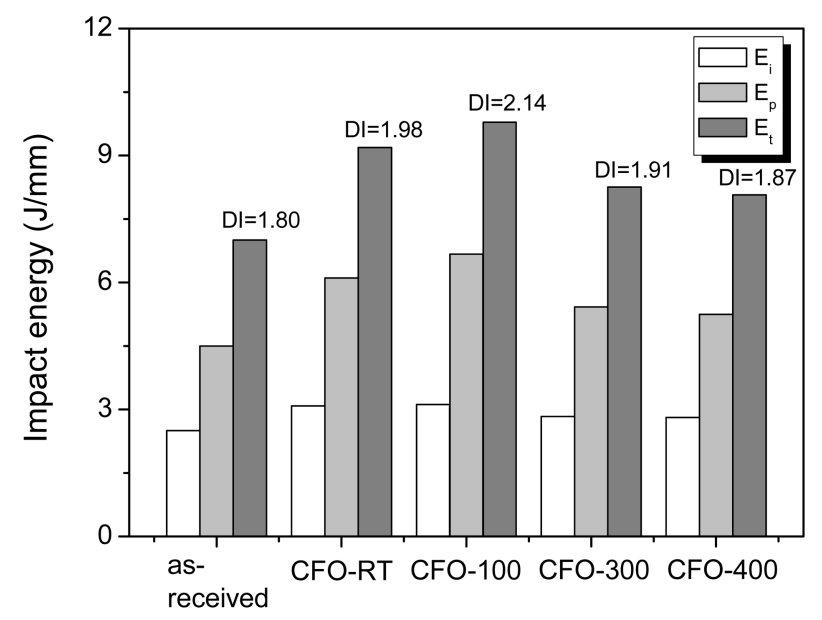

Figure 6. Impact energy of oxyfluorinated carbon fibers-reinforced composites as a function of oxyfluorination temperature. composites are shown in Figure 6. The total energy absorbed during the impact test, that is, both the impact energy and the $D I$ of the oxyfluorinated carbon fibers-reinforced composites increases with increasing fluorine concentration. This is probably due to the increased highimpact stiffness, resulting in enhanced ductile behavior, which can improve the entire range of the mechanical properties of such oxyfluorinated carbon fiber-reinforced composites.

\section{Conclusions}

In the present study, quasi-static, low-hanging, low-velocity impact tests were conducted to study the effect of fiber/ matrix adhesion on the impact properties of oxyfluorinated carbon fibers-reinforced epoxy matrix composites. The results indicated that the oxygen functional groups, $\mathrm{C}-\mathrm{O}, \mathrm{C}=\mathrm{O}$, and $\mathrm{HO}-\mathrm{C}=\mathrm{O}$ were present on the carbon fibers surfaces after oxyfluorination. According to an analysis of XPS results, the increased oxygen content improved the impact properties of the composites. In other words, the oxyfluorinated carbon fibers-reinforced composites, compared with the composites lacking oxyfluorination, much improved the impactdamage conditions, which is to say that the impact-loadinginduced failure behaviors were hindered by the effective hydrogen bonding between the fiber surfaces and the matrix resins in the composites.

\section{References}

(1) P. Ehrburger and J. B. Donnet, in Surface Treatment of Carbon Fibre for Resin Matrices, Strong Fibres: Handbook of Composites, Elsevier, New York, 1985.

(2) S. J. Park and Y. S. Jang, J. Colloid Interf. Sci., 237, 91 (2001).

(3) N. Iwashita, E. Psomiadou, and Y. Sawada, Composites Part A, 29, 965 (1998).

(4) J. Seo, W. Jang, and H. Han, Macromol. Res., 15, 10 (2007).

(5) W. Ruland, Adv. Mater., 2, 528 (1990).

(6) H. T. Ham, C. M. Koo, S. O. Kim, Y. S. Choi, and I. J. Chung, Macromol. Res., 12, 384 (2004).

(7) J. M. Park and J. W. Kim, Macromol. Res., 10, 24 (2002).

(8) H. S. Lee and D. W. Cho, Macromol. Res., 16, 411 (2008).

(9) J. S. Park, J. M. Kim, S. J. Lee, S. G. Lee, Y. K. Jeong, S. E. Kim, and S. C. Lee, Macromol. Res., 15, 424 (2007).

(10) Y. S. Park and K. H. Chung, Elastomer, 42, 75 (2007).

(11) S. J. Park and K. D. Kim, Carbon, 39, 1741 (2001).

(12) T. Ramanathan, A. Bismarck, E. Schulz, and K. Subramanian, Compo. Sci. Technol., 61, 599 (2001).

(13) B. J. Kim, Y. S. Lee, and S. J. Park, Current Appl. Phys., 8, 736 (2008).

(14) W. S. Shin, S. J. Park, C. Park, and K. Kim, Macromol. Res., 15, 671 (2007).

(15) S. M. Choi, E. K. Lee, and S. Y. Choi, Elastomer, 43, 147 (2008).

(16) D. Sarmeo, S. Blazewicz, M. Mermoux, and Ph. Touzain, 
Carbon, 39, 2049 (2001).

(17) K. G. Saw and J. du Plessis, Mater. Lett., 58, 1344 (2004).

(18) R. A. Levy, L. Chen, J. M. Grow, and Y. Yu, Mater. Lett., 54, 102 (2002).

(19) M. O. W. Richardson and M. J. Wisheart, Composites Part A, 27, 1123 (1996)

(20) W. L. Lee and C. F. Lin, Mater. Sci. Eng. A, 308, 124 (2001).
(21) S. J. Park, Y. S. Jang, and K. Y. Rhee, J. Colloid Interf. Sci., 245, 383 (2002).

(22) D. V. Bucci, M. J. Koczak, and L. S. Schadler, Carbon, 35, 235 (1997).

(23) V. K. Srivastava, K. Maile, and A. Klenk, Mater. Sci. Eng. A, 271, 38 (1999). 